\title{
Present status and future prospects of nEDM experiment of PNPI-ILL-PTI collaboration
}

\author{
Anatolii Serebrov ${ }^{1}$ \\ Petersburg Nuclear Physics Institute NRC KI \\ Gatchina, Russia \\ E-mail: serebrov_apepnpi.nrcki.ru
}

Use of ultracold neutrons (UCN) gives unique opportunities of a research of fundamental interactions in physics of elementary particles. Search of the electric dipole moment of a neutron (EDM) aims to test models of CP violation. Precise measurement of neutron lifetime is extremely important for cosmology and astrophysics. Considerable progress in these questions can be reached due to supersource of ultracold neutrons on the basis of superfluid helium which is under construction now in PNPI NRC KI. This source will allow us to increase density of ultracold neutrons approximately by 100 times in respect to the best UCN source at high flux reactor of Institute Laue-Langevin (Grenoble, France). Now the project and basic elements of the source are prepared, full-scale model of the source is tested, the scientific program is developed. Increase in accuracy of neutron EDM measurements by order of magnitude, down to level $10^{-27}-10^{-28} \mathrm{e} \mathrm{cm}$ is planned. It is highly important for physics of elementary particles. Accuracy of measurement of neutron lifetime can be increased by order of magnitude also. At last, at achievement of UCN density $\sim 10^{3}-10^{4} \mathrm{~cm}^{-3}$, the experiment search for a neutron-antineutron oscillations using UCN will be possible. The present status of the project and its scientific program will be discussed.

The 26th International Nuclear Physics Conference

11-16 September, 2016

Adelaide, Australia

\section{${ }^{1}$ Speaker}




\section{Experiment on search for neutron EDM}

Why is search for nEDM of great scientific importance? The standard Model (SM) does not explain symmetry violation between matter and antimatter in the Universe (everything around us consists of matter, while there is almost no antimatter). The supersymmetry theory is currently the most widely spread one, within the frame of which CP -symmetry is violated in the way that can account for baryon asymmetry of the Universe. One and the same mechanism of CP- symmetry violation creates $\mathrm{nEDM}$ and the Universe baryon asymmetry, therefore studying neutron, one can learn how asymmetry originated during baryon genesis at the Universe primordial stage.

A.D. Sakharov in 1967 [1] for the first time discovered that for interpretation of the Universe baryon asymmetry, one should assume that there exists the interaction which, firstly, does not conserve baryon number and, secondly, violates CP- invariance. Such interactions are beyond the Standard Model, thus the Universe baryon asymmetry interpretation is outside the scope of the Standard Model.

The experiment on search for neutron EDM started in PNPI at the beginning of the 1970s [2]. Russia has its priority in the discovery of ultracold neutrons and their employment for nEDM search [3,4]. Figure 1 shows in stages this experiment development in PNPI and in the ILL, as well as demonstrates the aim of the project - to attain accuracy of $3 \cdot 10^{-28} \mathrm{e} \cdot \mathrm{cm}$ at the UCN source with superfluid $\mathrm{He}$ at the reactor WWR-M [5].

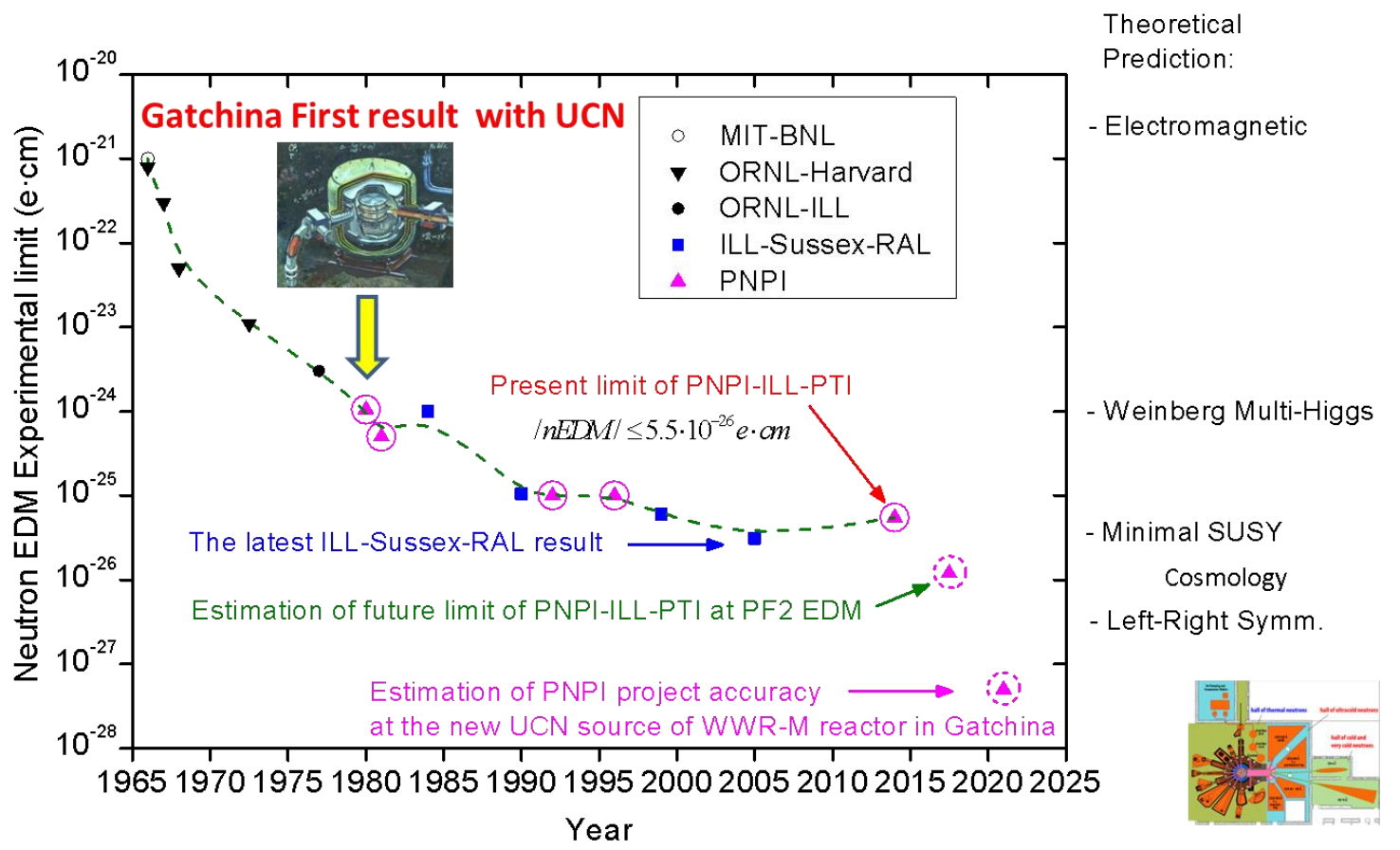

UCN source at WWR-M reactor

Figure 1. History of decreasing an experimental limit on neutron EDM and prospects for accuracy increase in PNPI NRC KI at the new source of UCN at the reactor WWR-M.

This tendency in research is currently actively developing in the world with obvious competition. In the1980s there were only two centers - PNPI and ILL which performed research on search for neutron EDM with UCN, while now there are as many as nine of them [6]. Accuracy of measurements is determined by the UCN source intensity. Hence, where the most intensive UCN source will be created, the most accurate result will be obtained and thus, nEDM is likely 
to be discovered. In high energy physics, the determining parameter is proton energy on colliders, whereas in our case, it is the UCN source intensity which is of primary importance.

Our goal is to create the UCN source with the highest possible intensity at the reactor WWR-M in PNPI NRC KI and to make measurements with accuracy of $3 \cdot 10^{-28} \mathrm{e} \cdot \mathrm{cm}$ (figure 1).

At present PNPI EDM spectrometer is modernized and is being prepared for transporting to a more intensive UCN beam PF2 EDM in ILL. The goal is to reach the accuracy of $1 \cdot 10^{-26} \mathrm{e} \cdot \mathrm{cm}$, i.e. 3 times exceeding the present limit [7]. However, the construction of our own UCN source of high intensity at the reactor WWR-M still remains the main task.

\section{UCN source}

The creation of this source started in 2006. Resource of the reactor tank allows its employment for another 25 years. WWR-M reactor is unique for creating the best in the world UCN source. This is determined by having a thermal column of a big size, where in neutron flux of $3 \cdot 10^{12} \mathrm{n} \cdot \mathrm{cm}^{-2} \mathrm{~s}^{-1}$ the UCN source on superfluid He can be installed. PIK reactor has apparent advantages for thermal and cold neutrons, however, for UCN, as shown below in detail, it is more preferable to employ WWR-M reactor. PNPI has a rich experience in developing and creating UCN sources (figure 2).

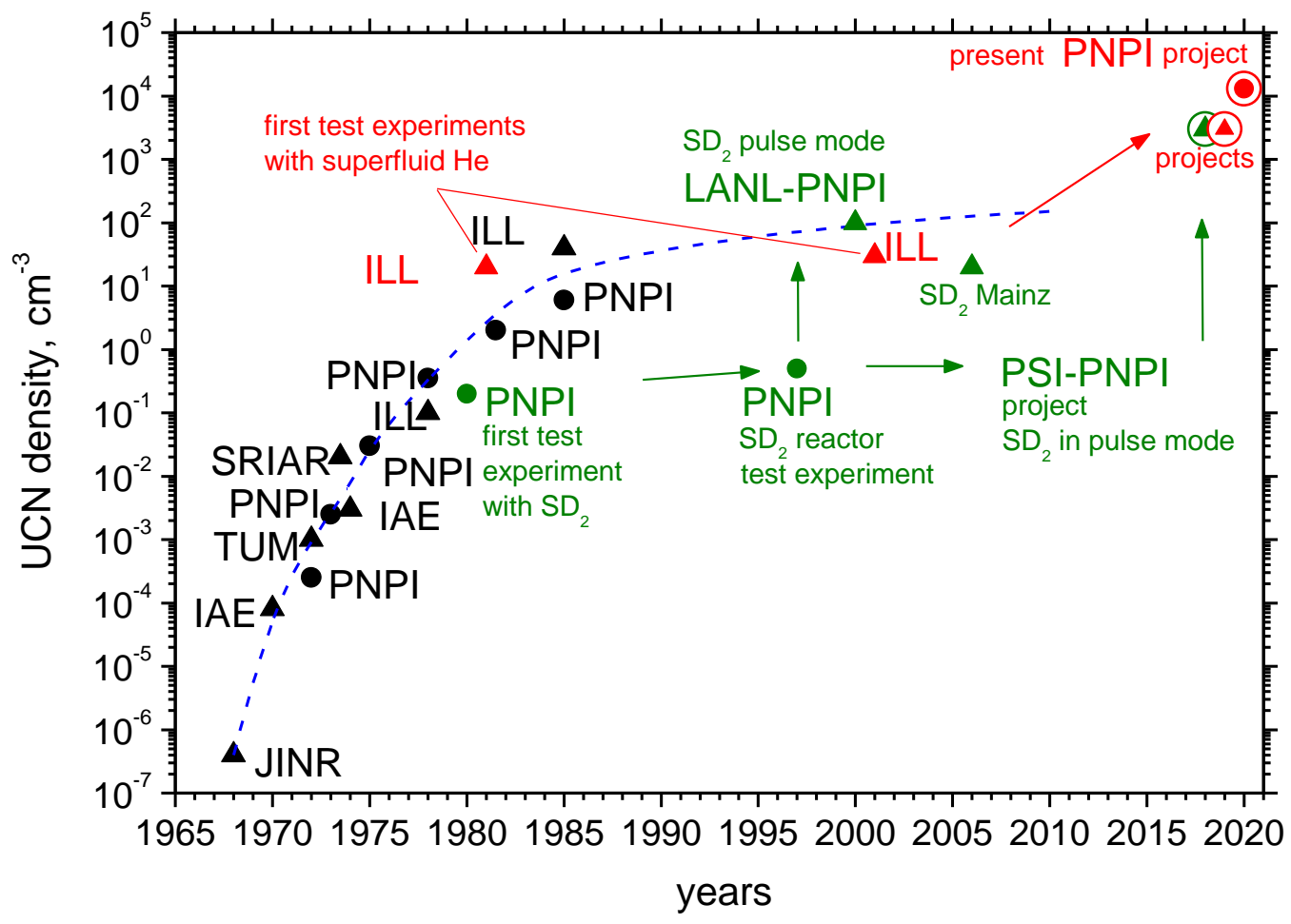

Figure 2. Progress in development of UCN sources. The last point in this diagram is related to project parameters of the new source at the WWR-M reactor PNPI on superfluid He with indicated possible UCN density in the EDM spectrometer trap. Other circles with triangles inside mark the following: the project on hard deuterium in Switzerland in PSI (intensity is so far by two orders of magnitude less than expected) and the ILL project on superfluid He and UCN magnetic trap (test experimental stage).

At present, PNPI NRC KI has elaborated the UCN source project for the reactor WWR-M. The project is based on using super fluid $\mathrm{He}$ in producing ultracold neutrons. The technique of 
applying super fluid He has been tested many times [8], and most new projects for UCN production are focused on it. PNPI NRC KI has currently created a full scale model of the source which involves all the required cryogenic and vacuum equipment. For time being, using the full scale model we have succeeded in maintain $\mathrm{He}$ in super fluid state at temperature $1.3 \mathrm{~K}$ with thermal loading of $50 \mathrm{~W}$.

It could be easily assumed that in future one can obtain even higher UCN density for the experiment on search for neutron EDM at high flux reactor PIK. However, a detailed consideration of the problem shows that this is not the case. This question is of great importance for us so the detailed study was performed and we believe it worth further discussion. The reactor PIK is suitable for thermal and cold neutrons, however, WWR-M reactor suits better for UCN due to availability of a thermal column.

The difference is that at the reactor PIK the UCN source on superfluid He can be located only on the extracted neutron beam. Yet, transition to the UCN source scheme at the extracted beam results in loss of the initial density of neutron flux proportionally to the beam solid angle with respect to $4 \pi$. At the distance of 5 meters from the source of cold neutrons the solid angle factor is $10^{-4}$. To compensate for such a factor is extremely difficult. The neutron flux in the thermal column of the WWR-M reactor is $3 \cdot 10^{12} \mathrm{n} \cdot \mathrm{cm}^{-2} \mathrm{~s}^{-1}$, and the neutron flux in the source of cold neutrons of the reactor PIK is approximately (3-5) $\cdot 10^{14} \mathrm{n} \cdot \mathrm{cm}^{-2} \mathrm{~s}^{-1}$. Thus, in transition to the reactor PIK on the UCN source scheme, four orders of magnitude are lost at the extracted beam because of the beam solid angle, but one succeeds in compensating only two orders of magnitude on account of a high neutron flux in the PIK reactor. The total UCN output of the source can be compensated a few times by increasing 5 times the convertor volume with superfluid He, locating it along the beam of cold neutrons. However, it will be enhancement of the total output rather than density of UCN production in the source.

Hence, with EDM spectrometer trap it is possible to obtain UCN density of $1.3 \cdot 10^{4} \mathrm{~cm}^{-3}$ at UCN source in the thermal column of WWR-M reactor, while at the reactor PIK the UCN density is equal to $1.3 \cdot 10^{3} \mathrm{~cm}^{-3}$. Thus, the UCN source in the thermal column of the WWR-M reactor is 10 times more efficient [9]. Finally, it should be noted that the UCN source for the reactor WWR$\mathrm{M}$ is to a great extent completed, whereas perspectives for the reactor PIK are not determined yet.

Figure 3 shows the beam scheme of the reactor WWR-M after installing UCN source. Figure 4 presents the location scheme of cryogenic and experimental equipment to be implemented in 3 years at the WWR-M reactor. Essentially, it is an overall experimental complex. At present all the cryogenic equipment and experimental installations are available for it. However, cryogenic equipment (positions 4-7) is now accommodated in the cryogenic hall of the first building near the cryogenic facilities (positions 8-13). This is required for conducting a full-scale test experiment, i.e. testing the possibility to remove thermal power and to maintain temperature of 1.2$1.3 \mathrm{~K}$. After a successful test, cryogenic equipment should be dismantled in accordance with the scheme in figure 5. Intra-channel part of the source has been projected.

Our project will be a rather successful advancement in conservation and development of fundamental and applied research in Russia on the basis of research reactors. The project does not require any capital construction and can be implemented during 3-5 years. Owing to the developed reactor infrastructure the project concerned is economically high efficient. 


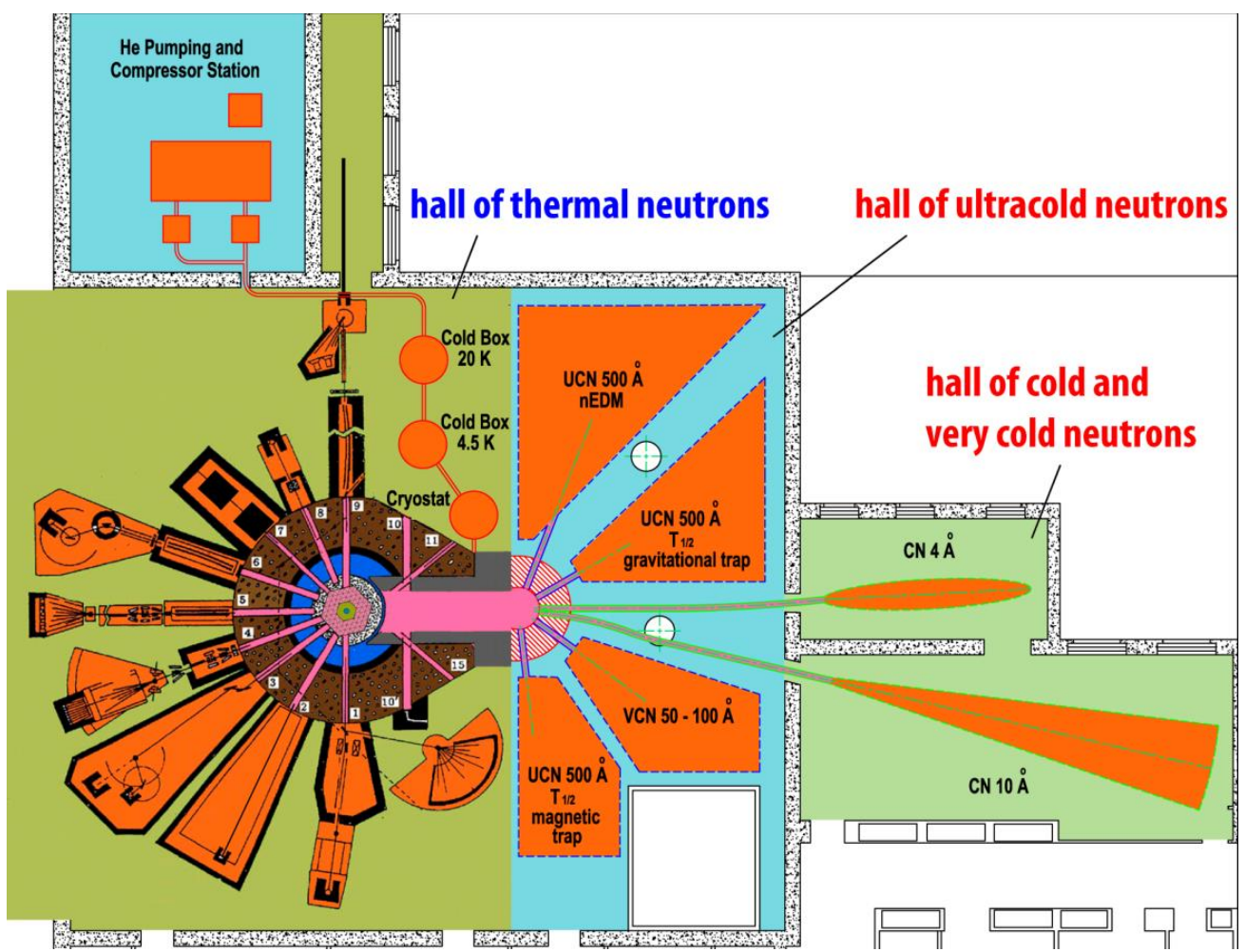

Figure 3. Experimental halls of the reactor WWR-M. The location scheme of the source of cold and ultracold neutrons, complex of experimental installations in the main reactor hall and complex of experimental installations in neutron guide halls.

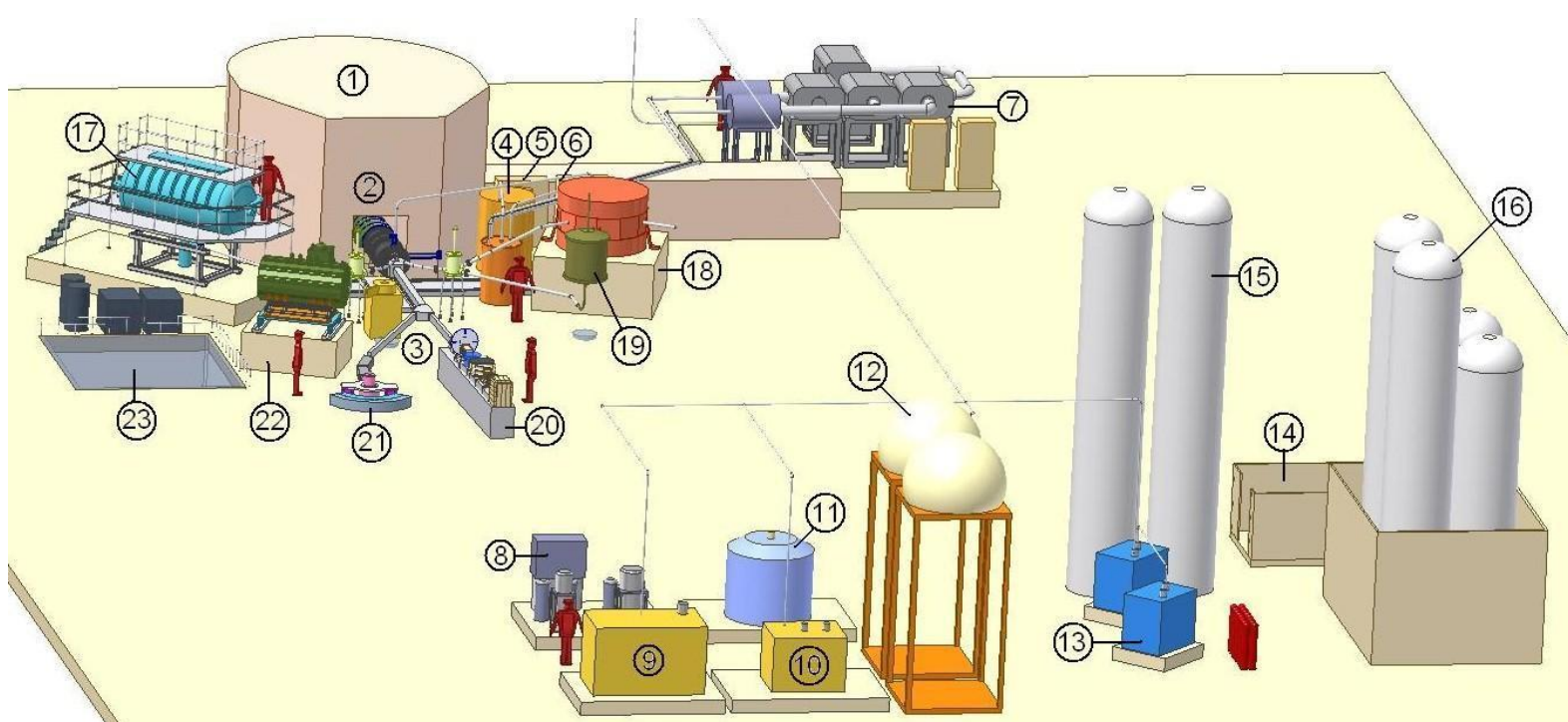

Figure 4. Complex of facilities and experimental installations on the reactor WWR-M. 1. WWR-M reactor. 2. Intra-channel part of UCN source in the thermal column of the reactor. 3. Neutron guide system. 4. The cryostat for superfluid helium. 5. He refrigerator on 15K. 6. He liquefier. 7. System of vacuum pumping. 8. Cleaning block He. 9. Compressor for refrigerator. 10. Compressor for He liquefier. 11. He dewar. 12. He gas-holder. 13. Downloading compressors He in cylinders. 14. Balloon cell. 15. He receivers. 16. D2 receivers. 17. Gravitational trap for measurement of neutron lifetime. 18. EDM spectrometer. 19. A magnetic trap for measurement of neutron lifetime. 20. Reflectometer. 21. Spin-echo a spectrometer with VCN. 22. Installation for search of mirror dark matter. 23. WWR-M reactor ramp. 


\section{Scientific program for UCN source at the reactor WWR-M}

Figure 5 gives the location scheme of experimental installations for operation with UCN. Besides the apparatus for measuring nEDM, two installations for neutron lifetime measurement are presented: one with a big gravitational trap (Gravitrap) and another one with a magnetic trap (magnetic trap). Precision estimations of neutron lifetime are important for verification of the model of the Universe formation at its early stage as well as for search for deviations from the Standard Model. In addition to it, an installation for search for mirror dark matter is presented ( $\mathrm{n}-$ n'). All these installations have been created and tested at UCN beams in ILL. They will be transferred to the PNPI new source of UCN. The UCN intensity enhancement by more than two orders of value will enable to carry out principally new research. Finally, one can discuss performance of an experiment on search for neutron-antineutron oscillations (n- $\tilde{n})$ for highly intensive UCN source with the aim of testing violation of baryon number, i.e. the second condition for the Universe origin according to A.D. Sakharov theorem.

Thus, besides the crucial experiment on search for neutron EDM, there arise opportunities for carrying on the whole series of experiments on physics of fundamental interactions.

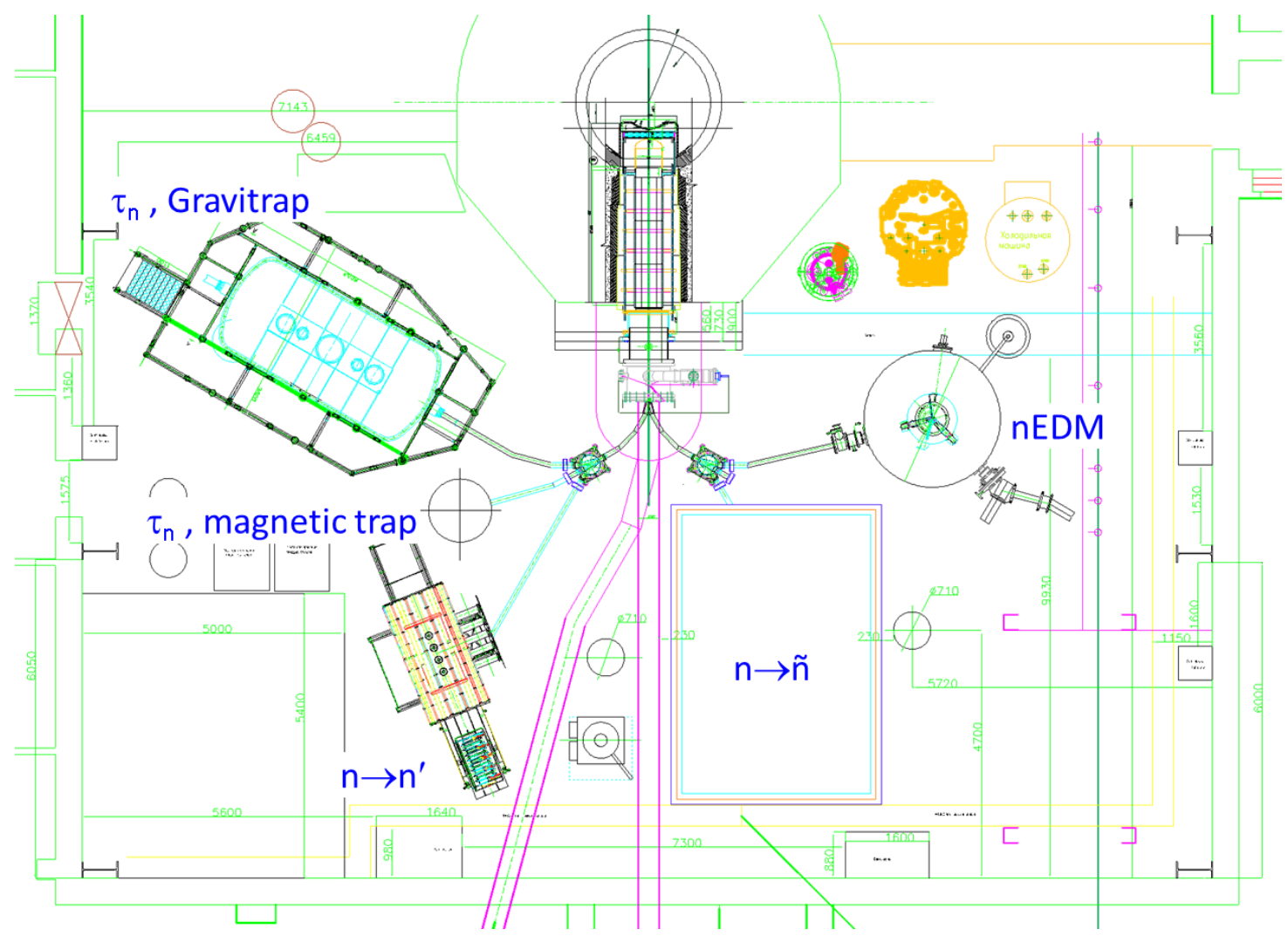

Figure 5. Scheme of experimental apparatus location in the hall of ultracold neutrons of the WWR-M reactor.

Precision measurements of neutron lifetime are crucial for testing the Universe formation model at its early stage as well as for search for deviations from the Standard Model. Precision neutron lifetime measurement gives experimental data for calculation of cross section of reverse beta decay reaction. This is extremely important for calculation of efficiency of neutrino detectors. 


\subsection{Neutron lifetime measurement using gravitational UCN trap}

The first experiment with a gravitational trap of small volume has obtained the result for neutron lifetime $878.5 \pm 0.8 \mathrm{~s}$ [10]. The novel experiment with a big gravitational trap is on the mounting stage at the reactor in ILL. Principle of gravitational shutter for holding UCN in a material trap is applied in the installation. Increase of precision is essential for investigating inconsistency outlined between different approaches to estimation of neutron lifetime - the beam approach and the UCN storage one.

Thus, estimation of time of UCN storage in a magnetic trap is the third independent and very important method of measurement.

\subsection{Neutron lifetime measurement by UCN storage technique in a magnetic trap}

Possibility of neutron storage in magnetic traps was discussed in detail at the very outset of their discovery by V.V. Vladimirsky (ITEP NRC KI) [11].

Modern technology enables creating permanent magnets with saturation induction of $1.4 \mathrm{~T}$ and higher. On the basis of such magnets the laboratory of V.F. Ezhov in PNPI NRC KI in collaboration with the group from Technical University of Munich and the Institute Laue-Langevin (Grenoble) created a magnetic trap with magnetic field on the wall of the order of magnitude of $1 \mathrm{~T}$.

In 2004-2005 it was tested at the UCN beam in ILL. During April - August 2005 the time of neutron holding in this trap was measured. The estimated value was $878.3 \pm 1.9 \mathrm{~s}$ [12]. This value approaches the period of neutron beta decay. No losses caused by depolarization of neutrons during their storage in the trap were found. Increasing the trap volume by 1 order will enable to attain accuracy of $0.3 \mathrm{~s}$.

\subsection{Neutron-antineutron oscillations}

One should note another possibility of using UCN in fundamental experiments. It is concerned with search for neutron-antineutron oscillations. Transition of neutron into antineutron is likely to occur only when the conservation law of baryon number is violated. As mentioned above, violation of the baryon number is one of the conditions for the Universe origin formulated by A.D. Sakharov. Study of this problem is no less essential than search for neutron EDM. At present there is a limit on the time of neutron-antineutron oscillations of $8.6 \cdot 10^{7} \mathrm{~s}$ [13], which was attained in the experiment with cold neutrons. Employing ultracold neutrons can result in raising sensitivity of the experiment in case of considerable enhancement $\left(10^{2}-10^{3}\right.$ times $)$ of the ultracold neutron density [14].

At present a conceptual project for the experiment on search for neutron-antineutron oscillations with the help of UCN is prepared.

\subsection{Search for mirror dark matter}

Due to violation of space invariance in weak interaction our world turned out to be the left. The reason for such a disparity of the right and the left is unknown. The Standard Model successfully explains how weak interaction is organized but it does not explain why the left (V-A) variant of the theory was chosen. Theoretically, if there is left asymmetry, why there could not be the right one, i. e. its mirror representation. Such reasoning might lead to far-reaching consequences 
on possibility of existence of mirror particles, mirror matter, etc. They come out to be even more urgent in relationship with existence of dark matter in the Universe, which is observed on account of peculiarities of gravitational phenomena and whose abundance is 5 times higher than that of ordinary matter. Sufficiently high activity in experimental testing of such hypothesis by neutron investigations arose in 2007. During 2007 three independent experiments on this problem were carried out. The most precise result was obtained by the collaboration of PNPI and ILL. Measurements were made in ILL at the UCN beam. Oscillation effect was not discovered, and the limit on oscillation time is $448 \mathrm{~s}$ [15] and the corresponding limit on the energy of mixing is $1.4 \cdot 10^{-18} \mathrm{eV}$.

UCN intensity enhancement by 2 orders of magnitude at the new source of the reactor WWR-M will provide considerable advancements in this research. An experimental installation for their performance is already available.

\section{Conclusion}

In conclusion, it is important to emphasize that techniques of precision investigations, those of search for small deviations from standard laws of physics allow to obtain information on fundamental interactions and to compete successfully with research on colliders. Examples of such investigations are presented in the program involved. Implementation of the experiment on search for neutron EDM with accuracy of $10^{-27} \mathrm{e} \cdot \mathrm{cm}$ and on search for oscillations is of great importance for physics of fundamental interactions.

At present in America (at NSS) and Europe (ILL, FRM-2, ESS) it is planned to carry out a few experiments on measuring neutron EDM, neutron lifetime and research on neutron decay asymmetry as well as on neutron - antineutron oscillations (ESS), therefore the program of PNPI NRC KI is in keeping with development of this tendency in physics. Investigations in this direction are performed in big competition which is determined by significance of this scientific problem. As mentioned above, intensity of the ultracold neutron source is a key issue in this competition. Investigations with ultracold neutrons started in Russia, so we must keep priority in this scientific direction. Creation of UCN source of high intensity at WWR-M reactor is a key issue in this strategy. Realization of the project with superfluid He at reactor WWR-M will make it possible to create in Russia the best in the world UCN source with intensity approximately 100 times exceeding that at the ILL reactor in Grenoble, the best international neutron center. With UCN source of high intensity the WWR-M reactor is expected to claim for becoming the international center of investigations on ultracold neutrons.

\section{Acknowledgments}

This work was carried out at Petersburg Nuclear Physics Institute NRC KI under the support of the Russian Science Foundation (project no. 14-22-00105).

\section{References}

[1] A.D. Sakharov 1967 JETP Lett. 524

[2] A.I. Egorov et al. 1975 Yad. Fiz. 21292

[3] V.I. Lushchikov et al. 1969 JETP Lett. 923 
[4] F.L. Shapiro 1968 Sov. Phys. Usp. 11345

[5] A.P. Serebrov 2011 Crystallogr. Rep. 561230

[6] P. Schmidt-Wellenburg 2016 arXiv:1602.01997

[7] C.A. Baker et al. 2006 Phys. Rev. Lett. 97131801

[8] R. Golub et al. 1983 Z. Phys. B 51187

[9] A.P. Serebrov et al. 2016 Crystallogr. Rep. 61144

[10] A. Serebrov et al. 2005 Phys. Lett. B 60572

[11] V.V. Vladimirskii 1961 Sov. Phys. JETP 12740

[12] V.F. Ezhov et al. 2014 arXiv:1412.7434

[13] M. Baldo-Ceolin et al. 1994 Z. Phys. C 63409

[14] A.P. Serebrov et al. 2016 Tech. Phys. Lett. 4299

[15] A.P. Serebrov et al. 2009 Nucl. Instr. Meth. A 611137 